\title{
Effect of Exercise on Perfusion of Collateral-Dependent Myocardium in Dogs with Chronic Coronary Artery Occlusion
}

\author{
Paul R. Lambert, David S. Hess, and Robert J. BaChe \\ From the Veterans Administration Hospital, Durham, North Carolina 27705, the Department \\ of Medicine, Duke University Medical Center, Durham, North Carolina 27710, and the Department \\ of Medicine, University of Minnesota Hospitals, Minneapolis, Minnesota 55455
}

A B S TRACT Since the ability of mature intercoronary collateral channels to increase myocardial blood flow in response to drug-induced coronary vasodilation has been questioned, the present study was undertaken to evaluate the response of coronary collateral circulation to the stress of exercise. Studies were performed at rest and during two levels of treadmill exercise in six dogs a minimum of 6 mo after placement of an Ameroid constrictor on the left circumflex coronary artery. Regional myocardial blood flow was estimated in normally perfused anterior and predominantly collateral-dependent posterior left ventricular wall with left atrial injections of radionuclide-labeled microspheres $7-10 \mu \mathrm{m}$ in diameter. At rest, heart rate was $87 \pm 7$ beats/min and mean myocardial blood flow was comparable in control and collateral-dependent regions $(0.96 \pm 0.13$ and 0.97 $\pm 0.14 \mathrm{ml} / \mathrm{min} \cdot \mathrm{g}$, respectively). During exercise, heart rates increased to $180 \pm 13$ and $228 \pm 14$ beats/min and myocardial blood flow (MBF) in the anterior control region increased linearly with heart rate (HR), $(\mathrm{MBF}=0.133 \mathrm{HR}-0.202, r=0.88) . \mathrm{MBF}$ to the posterior collateral-dependent region was similarly augmented during exercise $(\mathrm{MBF}=0.140 \mathrm{HR}-0.252$, $r=0.89$ ), so that the linear correlation between HR and $\mathrm{MBF}$ was similar for the control and collateral-

This work was presented, in part, at the 48th Scientific Session of the American Heart Association, Anaheim, Calif., November 1975.

Mr. Lambert is a Senior Medical student, Duke University Medical Center. Dr. Bache is recipient of Research Career Development Award 1-KO4-HL-00039 from the U.S. Public Health Service.

Reprint requests should be addressed to Dr. Robert J. Bache, University of Minnesota Hospitals, Minneapolis, Minn. 55455.

Received for publication 23 June 1975 and in revised form $\$ 2$ September 1976. dependent regions. In addition, the transmural distribution of $\mathrm{MBF}$ was uniform at rest and during exercise in both the anterior control and posterior collateral-dependent regions. Thus, not only could the mature intercoronary collateral vasculature supply adequate flow at rest, but when subjected to the natural stress of exercise, the increase in flow to the predominantly collateral-dependent area was similar to that in the normally perfused area.

\section{INTRODUCTION}

Substantial evidence has been accumulated to demonstrate the rapid growth of intercoronary collateral vessels in response to gradual coronary artery occlusion in the dog. The functional capacity of fully transformed collateral vessels, however, has not been clearly defined. By quantitating total collateral inflow and transmural distribution, it has been demonstrated that during quiet resting conditions there is no difference in myocardial perfusion by the well-developed collateral circulation and the normal coronary circulation (1-4). In contrast to the resting state, however, it has been shown that during pharmacologically induced coronary vasodilatation or atrial pacing, blood flow to collateral-dependent regions does not increase as much as flow to normally perfused areas $(1,3-5)$. It was questioned whether a similar deficit in perfusion of collateral-dependent myocardium would occur during the coronary vasodilatation and tachycardia of exercise. Consequently, the present study was performed to measure regional myocardial perfusion and transmural distribution of myocardial blood flow during exercise in dogs with Ameroid constrictors chronically implanted on the proximal left circumflex coronary artery. To ensure that a mature collateral circulation was being assessed, a minimum of 6 mo was allowed 
from the time of constrictor implantation to the experimental intervention.

\section{METHODS}

Eight adult mongrel dogs weighing $22-34 \mathrm{~kg}$ were chosen for their ability to run on a motor-driven treadmill (model 1849-D, Quinton Instruments, Seattle, Wash.). After anesthesia with sodium thiamylal $(20-30 \mathrm{mg} / \mathrm{kg})$ and ventilation by a Harvard respirator (Harvard Apparatus Co., Inc., Millis, Mass.) a left thoracotomy was performed in the fourth intercostal space. The proximal $1.5 \mathrm{~cm}$ of the circumflex branch of the left coronary artery was dissected free and an Ameroid constrictor (Threepoint Products, Montreal, Quebec, Canada), chosen to fit snugly in contact with the artery, was placed around the vessel proximal to any branches (6). All animals survived this surgery, but two animals subsequently died, one 17 days and one 21 days postoperatively. Dogs were maintained on a normal laboratory diet and were allowed to exercise in open runs ad libitum. 6-10 mo (mean $=8 \mathrm{mo}$ ) after the initial surgery, the six surviving animals underwent a second left thoracotomy and a heparinfilled polyvinyl chloride catheter, $3 \mathrm{~mm} O \mathrm{OD}$, was inserted into the left atrial cavity through the atrial appendage and secured with a purse-string suture. A similar catheter was introduced into the arch of the aorta via the left internal mammary artery. Both catheters were tunneled to a subcutaneous pouch at the base of the neck but were not exteriorized to protect them from damage.

7-10 days after the second surgical procedure, a brief retraining period of 1-2 days was begun on the treadmill. Studies were performed 9-29 days after the second surgery when the dogs were free from fever, anemia, or other evidence of ill health. On the morning before study, the left atrial and aortic catheters were exteriorized from the subcutaneous pouch using $2 \%$ lidocaine infiltration anesthesia. The arterial catheter was attached to a Statham $\mathrm{P} 23 \mathrm{Db}$ pressure transducer (Statham Instruments Div., Gould Inc., Oxnard, Calif.) mounted on the dog's side and to a polyethylene tube connected to a withdrawal pump (model 1210, Harvard Apparatus Co., Inc.). The left atrial catheter was connected via a polyethylene tube to a Statham P23Db transducer positioned on the treadmill cage at the level of the left atrium. Four ECG needle electrodes were placed intradermally on the dog's ventral thorax and secured by sutures. Mean aortic and left atrial pressures and the electrocardiogram were recorded continuously on a direct-writing oscillograph (model 7700, Hewlett-Packard Co., Palo Alto, Calif.). Electrocardiograms obtained during exercise were adequate to exclude cardiac arrhythmias, but not to exclude ST or T-wave changes.

Measurements of regional myocardial blood flow were made by injecting into the left atrium carbonized microspheres (Nuclear Products Division, 3M Co., St. Paul, Minn.) 7-10 $\mu \mathrm{m}$ in diameter labeled with gamma-emitting radionuclides ${ }^{1+1} \mathrm{Ce},{ }^{51} \mathrm{Cr},{ }^{85} \mathrm{Sr}$, or ${ }^{46} \mathrm{Sc}$. The microspheres were diluted in $10 \%$ low molecular weight dextran so that $1.0 \mathrm{ml}$, the volume injected, contained approximately 3 million microspheres. Before each injection, the microspheres were mixed by alternate agitation for at least $15 \mathrm{~min}$ in an ultrasonic bath and vortex agitator. Complete dispersion was verified by microscopic examination of a drop of the microsphere suspension. Myocardial blood flow was measured during resting control conditions while standing, and during two levels of exercise on the treadmill, designated light and moderate. Light exercise was defined as the speed and grade (mean, $4.5 \mathrm{mph}, 4 \%$ grade) necessary to increase heart rates approximately 90 beats/min above the resting heart rate, while moderate exercise (mean, $6 \mathrm{mph}, 8 \%$ grade) was that level required to achieve heart rates of approximately 140 beats $/ \mathrm{min}$ above the resting rate. Two dogs were studied with only one level of exercise in addition to the resting state, this level being light in one case and moderate in the other. During each intervention, $1.0 \mathrm{ml}$ of the microsphere suspension was injected over a 5-s interval into the left atrium. The left atrial catheter was then flushed with $10 \mathrm{ml}$ of isotonic saline. Each microsphere injection was made $60 \mathrm{~s}$ after a hemodynamic steady state had been achieved $(90-120 \mathrm{~s}$ after the onset of exercise), as monitored by heart rate and arterial pressure. This 60-s time interval was chosen since previous investigators have shown that heart rate and coronary artery blood flow reach a steady state $10-20 \mathrm{~s}$ after the onset of exercise $(7,8)$. Beginning simultaneously with each injection and continuing for $90 \mathrm{~s}$, a reference sample of arterial blood was collected from the aortic catheter at a constant rate of 15.0 $\mathrm{ml} / \mathrm{min}$ with the withdrawal pump.

After completing the study, the dog was anesthetized with sodium thiamylal and sacrificed with a lethal dose of potassium chloride. The heart was removed and fixed in $10 \%$ buffered formalin. The mean heart weight was $208 \pm 8 \mathrm{~g}$ and mean left ventricular weight was $121 \pm 6 \mathrm{~g}$. The circumflex branch of the left coronary artery was sectioned at the site of the Ameroid constrictor and was noted to be completely occluded in each instance. The aorta, atria, right ventricle, epicardial fat, and large surface vessels were dissected from the left ventricle and discarded. The left ventricle was then sectioned into four transverse rings of approximately equal thickness parallel to the mitral valve ring, as previously described (9). The two central sections, rings 2 and 3, which comprised $57 \pm 1 \%$ of the left ventricular weight were used for study. Two full-thickness tissue blocks encompassing approximately $1.5 \mathrm{~cm}$ of the outer circumference of these two rings were removed, one from the anterior ventricular wall in the region including the anterior papillary muscle, and one from the posterior ventricular wall in the region including the posterior papillary muscle. Since injections of methylene blue into the circumflex coronary artery demonstrated myocardial staining within a distribution encompassing the posterior papillary muscle tissue blocks, these specimens were taken to represent the collateral-dependent myocardial regions, while the corresponding anterior papillary muscle specimens served as controls (10). Each tissue block was then sectioned into four equal transmural layers from the epicardial to the endocardial surface, weighed, and placed in vials for counting. These layers will subsequently be referred to as layers 1 to 4 , layer 1 being the most epicardial and layer 4 the most endocardial.

The myocardial and blood reference samples were counted in a gamma spectrometer with window settings selected to correspond to the peak energies of each nuclide. A digital computer was used to correct the counts per minute recorded in each window for background activity and for contaminant activity contributed by the accompanying isotopes. Knowing the rate of withdrawal of the reference sample $\left(F_{r}\right)$ and the radioactivity in the reference sample $\left(C_{r}\right)$, myocardial sample radioactivity $\left(C_{m}\right)$ was used to compute myocardial blood flow $\left(F_{m}\right)$ by the formula:

$$
F_{m}=F_{r} \cdot C_{m} / C_{r} .
$$

Each sample blood flow was then divided by the sample weight and expressed as milliliters per minute per gram of myocardium.

The regional myocardial blood flow to rings 2 and 3 was analyzed with multiple paired $t$ tests comparing each region and layer in ring 2 with the corresponding specimen in ring 3 . The $P$ values were adjusted by the Bonferonni inequality 
TABLE I

Hemodynamic Data

\begin{tabular}{|c|c|c|c|c|c|c|c|c|c|}
\hline & \multicolumn{3}{|c|}{ Heart rate, beats/min } & \multicolumn{3}{|c|}{ Aortic pressure, $m m \mathrm{Hg}$} & \multicolumn{3}{|c|}{ Left atrial pressure, $\mathrm{mm} \mathrm{Hg}$} \\
\hline & Rest & $\begin{array}{l}\text { Light } \\
\text { exercise }\end{array}$ & $\begin{array}{l}\text { Moderate } \\
\text { exercise }\end{array}$ & Rest & $\begin{array}{l}\text { Light } \\
\text { exercise }\end{array}$ & $\begin{array}{l}\text { Moderate } \\
\text { exercise }\end{array}$ & Rest & $\begin{array}{c}\text { Light } \\
\text { exercise }\end{array}$ & $\begin{array}{c}\text { Moderate } \\
\text { exercise }\end{array}$ \\
\hline 1 & 102 & 195 & 234 & 85 & 98 & 120 & 5 & 5 & 5 \\
\hline 2 & 60 & 160 & 220 & 106 & 120 & 132 & 1 & 6 & 9 \\
\hline 3 & 78 & 155 & 200 & 90 & 105 & 122 & 2 & 3 & 3 \\
\hline 4 & 90 & 226 & 280 & 80 & 110 & 125 & 2 & 4 & 4 \\
\hline 5 & 90 & 165 & - & 77 & 115 & - & 5 & 5 & - \\
\hline 6 & 102 & - & 204 & 105 & - & 122 & 4 & - & 2 \\
\hline Mean & 87 & $180^{*}$ & $228^{*}$ & 91 & $110^{*}$ & $124^{*}$ & 3.3 & 4.8 & 4.6 \\
\hline$\pm \mathrm{SE}$ & 6.5 & 13.4 & 14.4 & 5.1 & 3.8 & 2.1 & 0.7 & 0.6 & 1.2 \\
\hline
\end{tabular}

Heart rate, mean aortic pressure, and mean left atrial pressure of all dogs studied at rest and during light and moderate exercise.

* Denotes $P<0.005$ in comparison with values at rest.

(11) which corrects for performing multiple tests on correlated data, i.e., each $P$ value was multiplied by the number of paired $t$ tests performed on each set of data and a $P$ value of $<0.05$ was required for statistical significance. Since no statistically significant difference in blood flow to corresponding regions in rings 2 and 3 was found, data from the two rings were combined for subsequent analysis. The transmural distribution of flow from endocardium to epicardium was analyzed by computing a collateral flow index for each transmural layer, obtained by dividing flow to each layer of the collateraldependent posterior papillary muscle region by flow to the corresponding anterior region. Mean regional blood flow for each intervention was determined by averaging the flow to all four layers. Regression equations for heart rate plotted against mean blood flow to anterior and posterior papillary muscle regions were determined. An analysis of covariance was performed to compare the slopes of the resultant anterior and posterior regression lines.

\section{RESULTS}

Table I displays the hemodynamic data during resting conditions and two levels of treadmill exercise from six dogs with Ameroid occlusion of the left circumflex coronary artery. During quiet resting conditions, heart rates ranged from 60 to 102 beats $/ \mathrm{min}$ and mean arterial pressures were 77-106 mm $\mathrm{Hg}$. During light exercise, heart rates were $155-226$ beats/min (mean increase from rest to light exercise $=93 \pm 11$ beats $/ \mathrm{min}$ ), and mean arterial pressures were $98-120 \mathrm{~mm} \mathrm{Hg}$. Heart rates during moderate exercise ranged from 200 to 280 beats $/ \mathrm{min}$ (mean increase from rest to moderate exercise $=141 \pm 15$ beats $/ \mathrm{min}$ ), and mean arterial pressures ranged from 120 to $132 \mathrm{~mm} \mathrm{Hg}$. Arterial pressure increased significantly from rest to light exercise, and underwent a further significant increase from light to moderate exercise $(P<0.01)$. Left atrial pressures ranged from 1 to $9 \mathrm{~mm} \mathrm{Hg}$ and did not change significantly during exercise.

Myocardial blood flow to the control and collateral- dependent regions and to each transmural layer increased linearly with heart rate (Table II, Fig. 1). Flow to the anterior control region increased $117 \pm 23 \%$ during light exercise and $212 \pm 21 \%$ during moderate exercise, as compared to the mean blood flow measured at rest. Flow to the posterior collateral-dependent region was similarly augmented $118 \pm 21 \%$ and 232 $\pm 25 \%$ during light and moderate exercise. Mean myocardial blood flow to the control and collateralized regions plotted against heart rate are shown in Fig. 2. For both the anterior and posterior regions there was a positive linear correlation between heart rate and blood flow ( $r=0.88$ and $r=0.89$, respectively). Analysis of covariance demonstrated that the slopes of the two regression equations were not significantly different $(P>0.3)$. Thus, total blood flow to the

TABLE II

Myocardial Blood Flow

\begin{tabular}{|c|c|c|c|c|c|c|}
\hline \multirow[b]{2}{*}{ Dog } & \multicolumn{3}{|c|}{ Anterior papillary muscle region } & \multicolumn{3}{|c|}{ Posterior papillary muscle region } \\
\hline & Rest & $\begin{array}{l}\text { Light } \\
\text { exercise }\end{array}$ & $\begin{array}{l}\text { Moderate } \\
\text { exercise }\end{array}$ & Rest & $\begin{array}{c}\text { Light } \\
\text { exercise }\end{array}$ & $\begin{array}{c}\text { Moderate } \\
\text { exercise }\end{array}$ \\
\hline & \multicolumn{6}{|c|}{$\mathrm{ml} / \mathrm{g} / \mathrm{min}$} \\
\hline $1^{*}$ & 1.18 & 2.52 & 4.13 & 1.26 & 2.62 & 4.50 \\
\hline $2 *$ & 0.60 & 1.78 & 2.16 & 0.70 & 2.00 & 2.55 \\
\hline 3 & 0.89 & 1.78 & 2.59 & 0.91 & 1.88 & 2.94 \\
\hline 4 & 1.43 & 2.24 & 3.59 & 1.41 & 2.18 & 3.38 \\
\hline $5 \ddagger$ & 0.91 & 2.07 & & 0.85 & 2.04 & \\
\hline 6 & 0.74 & & 2.50 & 0.70 & & 2.88 \\
\hline Mean & 0.96 & 2.08 & 2.99 & 0.97 & 2.14 & 3.25 \\
\hline$\pm \mathrm{SE}$ & \pm 0.13 & \pm 0.14 & \pm 0.34 & \pm 0.14 & \pm 0.14 & \pm 0.32 \\
\hline
\end{tabular}

Mean myocardial blood flow for each dog at rest and during light and moderate exercise.

* Arterial segment within the Ameroid constrictor recanalized.

$\ddagger$ Arterial segment within the Ameroid constrictor totally occluded. 

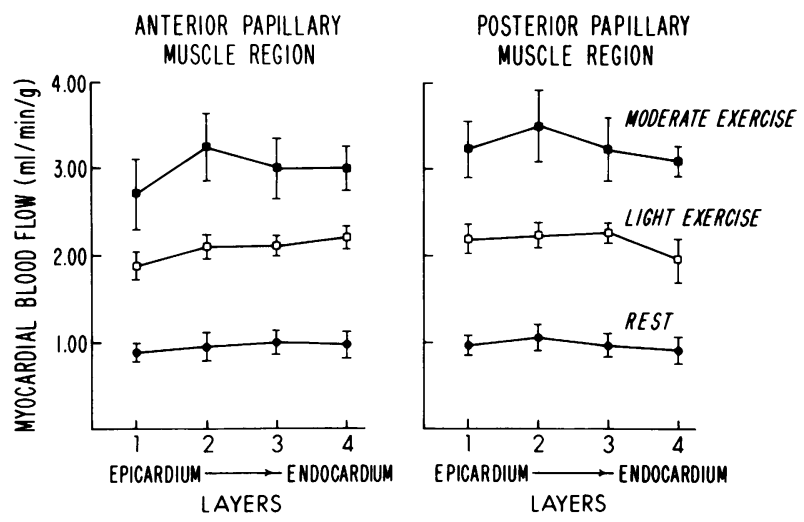

Figure 1 Mean blood flow \pm SEM to all transmural layers of the anterior and posterior papillary muscle regions of the left ventricle at rest and during light and moderate exercise.

collateralized area was comparable to flow in the normal myocardium both at rest and during exercise.

Transmural distribution of flow was examined by computing a collateral flow index for each transmural layer (Table III). This index was computed as the ratio of blood flow to the posterior collateral-dependent region divided by blood flow to the corresponding anterior control region. This ratio was not significantly different from 1.00 except in layer 1 , the most epicardial layer, during both light and moderate exercise, and in layer 2 at rest. In these cases, the collateral flows actually exceeded the control flows. Thus, resting blood flow and the augmented flow during exercise was uniformly distributed across the myocardial wall in the collateral-dependent region, as well as the normal myocardium.

Fig. 3 presents a comparison of the present data with results of a study previously reported from this laboratory in which the response of myocardial blood flow to graded treadmill exercise was examined in a group of nine control dogs with a normal coronary circulation (9). All the data points are represented in this graph, where posterior papillary muscle region flows are plotted against the corresponding anterior papillary muscle flows for each transmural layer in each of the six dogs at rest and during light and moderate exercise. It was found that 59 of the 64 data points fell within the $95 \%$ confidence limits defined in the previous study. Thus, blood flow to both the anterior and posterior papillary muscle regions of the dogs in the present study did not differ significantly from that observed in normal dogs $(P>0.3)$.

All hearts were examined for gross or microscopic evidence of infarction. In one heart (dog no. 5), an area of patchy subendocardial fibrosis was found in the posterior papillary muscle region, comprising approximately $10 \%$ of layer 3 and $40 \%$ of layer 4 . The resting blood flow in this region, compared to the correspond- ing anterior region, was similar in layer 3 but decreased $34 \%$ in layer 4 . During exercise, however, the percentage increase in blood flow for layers 3 and 4 was comparable in the posterior and anterior regions. This dog was included in the study since the decreased absolute flow did not significantly change the mean flow values for the group and since the ability to augment flow was not compromised by the presence of scar tissue.

\section{DISCUSSION}

The radionuclide-labeled microsphere technique has previously been shown to provide accurate and reproducible measurements of both total organ blood flow and blood flow to small regions of the left ventricular myocardium (12). Although this technique has been used extensively for study of the coronary collateral circulation, presumably with similar reliability $(1-5,13)$, it must be acknowledged that the precision with which microspheres estimate collateral blood flow has not been extensively evaluated. However, in comparison with other techniques (such as measurement of retrograde coronary pressure or flow, or accumulation or clearance of diffusible radioactive indicators), the microsphere technique offers several important advantages for assessment of the intercoronary collateral circulation in awake animals. First, the technique avoids the substantial technical complexities involved in obtaining measurements of retrograde flow or pressure distal to a coronary artery occlusion in awake animals. Second, because the microspheres are permanently trapped at the microvascular level, there is no necessity for rapid removal of the tissue to be analyzed as is the case with diffusible indicators. Finally, the availability of isotopes labeled with several gamma-emitting radionuclides allows for

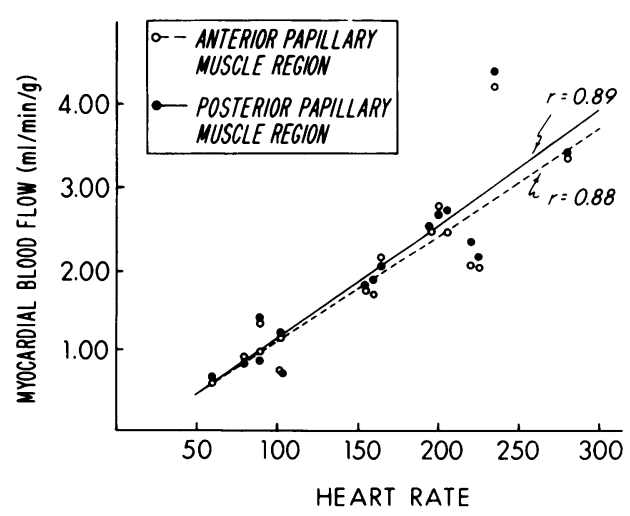

Figure 2 Mean blood flow to the anterior and posterior papillary muscle regions of the left ventricle plotted against heart rate for each dog at rest and during light and moderate exercise. 
TABLE III

Posterior Papillary Muscle/Anterior Papillary Muscle Flow Ratios

Transmural layers

\begin{tabular}{lcccc}
\hline & \multicolumn{4}{c}{ Transmural layers } \\
\cline { 2 - 5 } & 1 & 2 & 3 & 4 \\
\hline $\begin{array}{l}\text { Rest } \\
\begin{array}{c}\text { Light } \\
\text { exercise }\end{array}\end{array}$ & $1.13 \pm 0.07$ & $1.09 \pm 0.03^{*}$ & $0.97 \pm 0.05$ & $0.93 \pm 0.07$ \\
$\begin{array}{c}\text { Moderate } \\
\text { exercise }\end{array}$ & $1.23 \pm 0.08^{*}$ & $1.07 \pm 0.04$ & $1.08 \pm 0.05$ & $1.04 \pm 0.04$ \\
\hline
\end{tabular}

Ratios of posterior papillary muscle region blood flow divided by anterior papillary muscle region blood flow for each transmural layer measured at rest and during light and moderate exercise. Values are mean \pm SEM.

* Denotes $P<0.05$ in comparison with a ratio of 1.00 .

multiple determinations of blood flow within the same dog with each animal serving as its own control.

It has been repeatedly demonstrated that in the dog a small intercoronary collateral circulation is functional immediately after sudden coronary artery occlusion (13-17). Studies in which gradual coronary insufficiency was produced by an externally controlled constrictor cuff have demonstrated that these preexisting collateral vessels can undergo substantial development in a remarkably short time (18-20). One method used to explore the growth potential of intercoronary collateral channels has been the Ameroid constrictor model. Water absorption by the hygroscopic Ameroid material progressively narrows the artery lumen with total occlusion occurring in approximately $2^{1 / 2} \mathrm{wk}$, probably aided by connective tissue reaction in and around the stenosed vessel and by thrombosis (1). This method greatly reduces the incidence of myocardial infarction and the mortality from ventricular fibrillation which frequently accompanies acute coronary artery occlusion (1). In the present study, two of eight dogs died within 1 mo after Ameroid constrictor implantation, a time interval which encompassed the period of progressive stenosis and expected complete occlusion. Only one of the surviving six dogs developed myocardial infarction, indicating the substantial growth potential of a collateral circulation in response to gradual coronary artery occlusion. The occurrence of infarction may be explained by observations showing that for a short time before and after Ameroid occlusion, perfusion of the collateral-dependent myocardium may be inadequate (20).

The histology and time course of collateral vessel transformation after implantation of an Ameroid constrictor around the left circumflex coronary artery in dogs has been studied in detail by Schaper (1). He observed no further histologic change in the collateral vessel wall between 6 and 12 mo after Ameroid implantation, and concluded that the transformation process which resulted in a normal artery was completed within the initial 6-mo interval. Consequently, the dogs included in this paper were studied at a minimum of $6 \frac{1}{2}$ mo after implanting the Ameroid constrictor to ensure that a mature collateral circulation was being assessed.

Although the Ameroid occlusion model has been a widely used technique, systematic investigation of the possibility of incomplete coronary artery occlusion or recanalization of the occlusion has not been reported. The arterial segment within the Ameroid constrictor from three hearts in this study, all of which grossly appeared to be completely occluded, were subjected to serial sectioning and microscopic examination. One of these three specimens demonstrated total occlusion of the artery by dense fibrous tissue. Similar scar tissue was present in the arterial lumen of the other two specimens, but in each a recanalized lumen traversed the length of the Ameroid constrictor. In both of these hearts, the external diameter of the circumflex coronary artery was found to be $3.0 \mathrm{~mm}$; the diameters of the recanalized lumens were 0.35 and $0.23 \mathrm{~mm}$, respectively. Assuming the internal diameter of the original artery to be $2.0 \mathrm{~mm}$, these recanalized lumens represent cross-sectional areas of approximately 3.1 and $1.3 \%$ of the normal arterial lumen. The recanalized segment undoubtedly conducted antegrade flow into the region of myocardium distal to the Ameroid constrictor. It seems likely, however, that the contribution of this vessel would not be greater than that of two or three typical intercoronary collateral

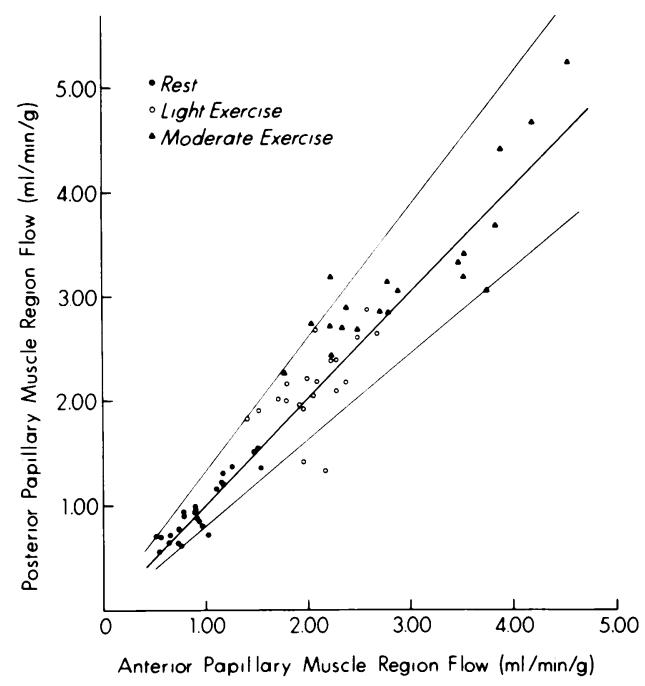

FIGURE 3 Posterior papillary muscle region blood flows are plotted against the corresponding anterior papillary muscle region blood flows for all transmural layers in each dog at rest and during light and moderate exercise. The $95 \%$ confidence limits obtained in a control study of nine dogs with normal coronary arteries are shown for comparison. 
vessels (generally $40-200 \mu \mathrm{m}$ in diameter), and functionally would merely add an additional collateral channel.

In contrast to the acute occlusion state in which collateral flow is small and nonuniformly distributed across the myocardial wall, the well-developed collateral circulation produced by Ameroid occlusion is adequate to perfuse its dependent myocardium during resting conditions (1-4). However it has been suggested that even in hearts with well-developed collateral vasculature, blood flow to collateral-dependent areas may be unable to increase normally and transmural perfusion may be altered (3). In open-chest dogs studied 3-6 wk after Ameroid occlusion had been documented by coronary angiography, Schaper et al. (3) found that endocardial/epicardial ratios of tracer microspheres were comparable in the posterior papillary muscle and anterior papillary muscle regions during resting control conditions (1.06 and 1.07, respectively). However, coronary vasodilation produced by administration of lidoflazine resulted in a highly significant transmural redistribution of blood flow in the collateral-dependent area, the endocardial/ epicardial flow ratio in the posterior left ventricular wall decreasing significantly to 0.78 , while in the anterior control region this ratio (1.03) did not change. Although lidoflazine increased flow to all areas of the heart, augmentation of flow to the collateral-dependent region was significantly less (1.70 times control flow) than to normally perfused areas (2.5 times control). Similar results have been reported with dipyridamole and norepinephrine $(1,4,5)$. In addition, atrial pacing 3 mo after Ameroid constrictor implantation has been shown to increase blood flow in normal myocardium, but to decrease flow in the subendocardium of the collateral-dependent area (4). It thus appeared that during pharmacologically induced coronary vasodilation, the collateral circulation could not augment flow as much as the normal coronary circulation, nor distribute it uniformly across the myocardial wall. The present study, however, did not reveal such a deficiency in total inflow or transmural distribution during the natural stress of exercise.

Why the collateral vasculature responded differently to the stresses of exercise and drug-induced coronary vasodilation is not immediately apparent. Factors which might influence total blood flow and transmural distribution to the collateral-dependent area include the degree of collateral maturity, coronary perfusion pressure, and the anesthetized, open-chest state. As previously described, the transformation process of the collateral vessel wall does not appear to be complete until approximately 6 mo after Ameroid implantation. Thus, it is possible that the 6-mo interval observed in the present study allowed the collateral vessels to achieve a greater potential for carrying blood than in previous studies in which pharmacologic vasodilators were administered 3-6 wk after Ameroid occlusion.

Availability of blood to the collateral-dependent myocardium could potentially be limited by the resistance to flow offered by the arteries which supply the collateral vessels. In the present study, heavy exercise increased flow to the collateral-dependent and control areas more than threefold, while Schaper observed flows after lidoflazine infusion that were 2.5 times control in the anterior papillary muscle region and only 1.7 times control in the posterior papillary muscle region. Since flows during exercise in the present study were substantially greater than those measured by Schaper et al. (3) during lidoflazine administration, it is unlikely that in Schaper's study the arteries supplying the collateral vessels had reached their maximum flow capacity. It is possible that the resistance to flow imposed by the collateral vessels could become significant if sufficient decrease in perfusion pressure occurred, however. Aortic pressure during administration of a coronary vasodilator may decrease significantly, while during exercise a significant increase in aortic pressure occurred. It is likely that the response of arterial pressure may be an important consideration when collateral blood flow is compared during coronary vasodilation produced by different mechanisms.

Even in the presence of a totally normal coronary circulation, the anesthetized, open-chest dog preparation may demonstrate nonuniform transmural myocardial perfusion during high flow states, especially in the distribution of the left circumflex coronary artery. Cobb et al. (12) observed that in open-chest dogs with normal coronary arteries, adenosine produced a fourfold average increase in total flow to the myocardium, but the subendocardial flow to the posterior wall and posterior papillary muscle regions increased significantly less than flow to the subendocardium of the anterior wall. Although the explanation for this is not clear, the data suggest that the response of the coronary vasculature to coronary vasodilator stimuli in the open-chest dog may differ from that in the awake dog.

This study has confirmed the ability of a welldeveloped coronary collateral circulation to perfuse its dependent myocardium during quiet resting conditions in the dog. More importantly, it demonstrated that during the natural stress of exercise, blood flow in all four of the transmural quarters of the predominantly collateral-dependent myocardium increased as much as in the normally perfused myocardium. Since the microsphere technique does not assess nutritional blood flow, however, the possibility that arteriovenous shunting may have occurred in the predominantly collateral-dependent area cannot be excluded. To result in microsphere trapping, however, such shunts would be required to have a functional diameter smaller than the microspheres $(7-10 \mu \mathrm{m})$. Neverthe- 
less, in the absence of biochemical data, the possibility that myocardial ischemia may have occurred in the collateral-dependent myocardium at some time during the exercise intervention cannot be unequivocally excluded. In addition, although this study revealed no inadequacy in the ability of the mature collateral circulation to perfuse its dependent myocardium, it does not exclude the possibility that perfusion deficits could occur with more strenuous exercise.

\section{ACKNOWLEDGMENTS}

The authors wish to acknowledge the continuing support of Dr. Joseph C. Greenfield, Jr. The following individuals rendered valuable assistance in carrying out this study: Dr. Judith C. Rembert, Kirby Cooper, Eric Fields, and James Ferrell; Donald G. Powell and the staff of the Durham Veterans Administration Hospital Medical Illustration Department; Michael Taylor and his staff of the Durham Veterans Administration Hospital Animal Care Facility; and Donna Silva and Rosa B. Ethridge, secretarial assistance.

This is Veterans Administration Program Number 0517-01. This work was supported in part by National Institutes of Health grant HL-20598 from the U.S. Public Health Service.

\section{REFERENCES}

1. Schaper, W. 1971. Collateral Circulation of the Heart. North-Holland Publishing Co., Amsterdam. 19-65.

2. Becker, L. C., and B. Pitt. 1971. Collateral blood flow in conscious dogs with chronic coronary artery occlusion. Am. J. Physiol. 221: 1507-1510.

3. Schaper, W., P. Lewi, W. Flameng, and L. Gijpen. 1973. Myocardial steal produced by coronary vasodilation in chronic coronary artery occlusion. Basic Res. Cardiol. 68: 3-20.

4. Pass, H. I., J. L. Cox, A. S. Wechsler, H. N. Oldham, Jr., and D. C. Sabiston, Jr. 1973. Response of coronary collateral circulation to increased myocardial demands. Circulation. 48 (Suppl. IV): 92. (Abstr.)

5. Schaper, W., W. Flameng, and M. DeBrabander. 1972. Comparative aspects of coronary collateral circulation. Adv. Exp. Med. Biol. 22: 267-276.

6. Litvak, J., L. E. Siderides, and A. M. Vineberg. 1957. The experimental production of coronary artery insufficiency and occlusion. Am. Heart J. 53: 505-518.

7. Khouri, E. M., D. E. Gregg, and C. R. Rayford. 1965.
Effect of exercise on cardiac output, left coronary flow and myocardial metabolism in the unanesthetized dog. Circ. Res. 17: 427-437.

8. Van Citters, R. L., and D. L. Franklin. 1969. Cardiovascular performance of Alaska sled dogs during exercise. Circ. Res. 24: 33-42.

9. Ball, R. M., R. J. Bache, F. R. Cobb, and J. C. Greenfield, Jr. 1975. Regional myocardial blood flow during graded treadmill exercise in the dog. J. Clin. Invest. 55: 43-49.

10. Bache, R. J., F. R. Cobb, and J. C. Greenfield, Jr. 1974. Myocardial blood flow distribution during ischemia-induced coronary vasodilation in the unanesthetized dog. J. Clin. Invest. 54: 1462-1472.

11. Miller, R. G., Jr. 1966. Simultaneous Statistical Inference. McGraw-Hill Book Co., New York. 67-69.

12. Cobb, F. R., R. J. Bache, and J. C. Greenfield, Jr. 1974. Regional myocardial blood flow in awake dogs. J. Clin. Invest. 53: 1618-1625.

13. Becker, L. C., N. J. Fortuin, and B. Pitt. 1971. Effect of ischemia and antianginal drugs on the distribution of radioactive microspheres in the canine left ventricle. Circ. Res. 28: 263-269.

14. Gregg, D. E., J. J. Thornton, and F. R. Mautz. 1939 Magnitude, adequacy and source of collateral blood flow and pressure in chronically occluded coronary arteries. Am. J. Physiol. 127: 161-175.

15. Kattus, A. A., and D. E. Gregg. 1959. Some determinants of coronary collateral blood flow in the openchest dog. Circ. Res. 7: 628-642.

16. Levy, M. N., E. S. Imperial, and H. Zieske, Jr. 1961. Collateral blood flow to the myocardium as determined by the clearance of rubidium ${ }^{86}$ chloride. Circ. Res. 9: $1035-1043$.

17. Cibulski, A. A., P. H. Lehan, and H. H. Timmis. 1972. Retrograde flow technique vs. krypton-85 clearance technique for estimation of myocardial collaterals. Am. J. Physiol. 223: 1081-1087.

18. Elliot, E. C., C. M. Bloor, E. L. Jones, W. J. Mitchell, and D. E. Gregg. 1971. Effect of controlled coronary occlusion on collateral circulation in conscious dogs. Am. J. Physiol. 220: 857-861.

19. Khouri, E. M., D. E. Gregg, and H. S. Lowensohn. 1968. Flow in the major branches of the left coronary artery during experimental coronary insufficiency in the unanesthetized dog. Circ. Res. 23: 99- 109.

20. Elliot, E. C., E. L. Jones, C. M. Bloor, A. S. Leon, and D. E. Gregg. 1968. Day-to-day changes in coronary hemodynamics secondary to constriction of circumflex branch of left coronary artery in conscious dogs. Circ. Res. 22: 237-250. 